\title{
The inner world of a collapsing bubble
}

\section{O. Supponen, P. Kobel, M. Farhat} EPFL - LMH, Lausanne, Switzerland

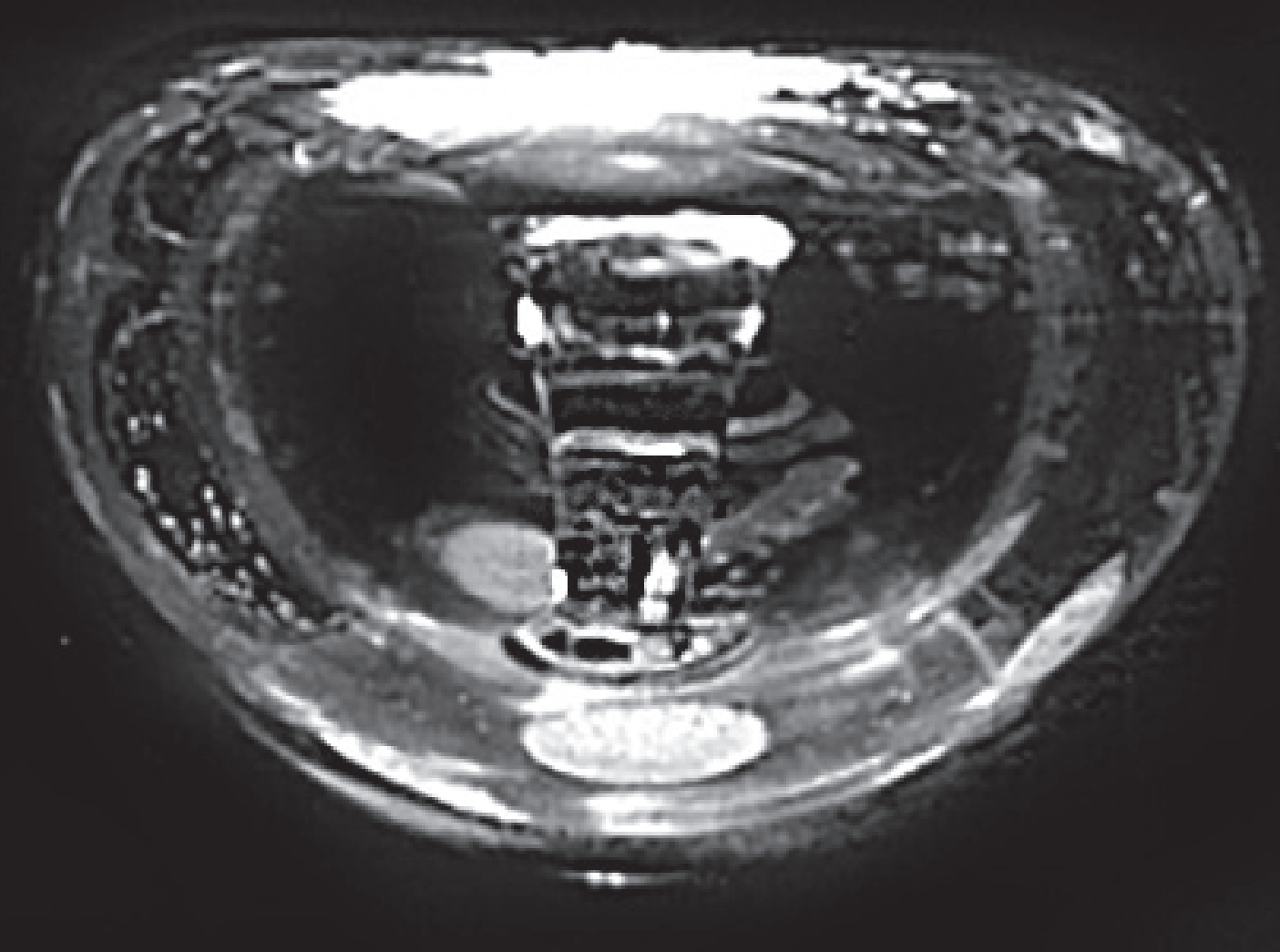

As the bubble expands nearby a free surface, it generates a pressure gradient between itself and the surface leading to a formation of a re-entrant microjet.

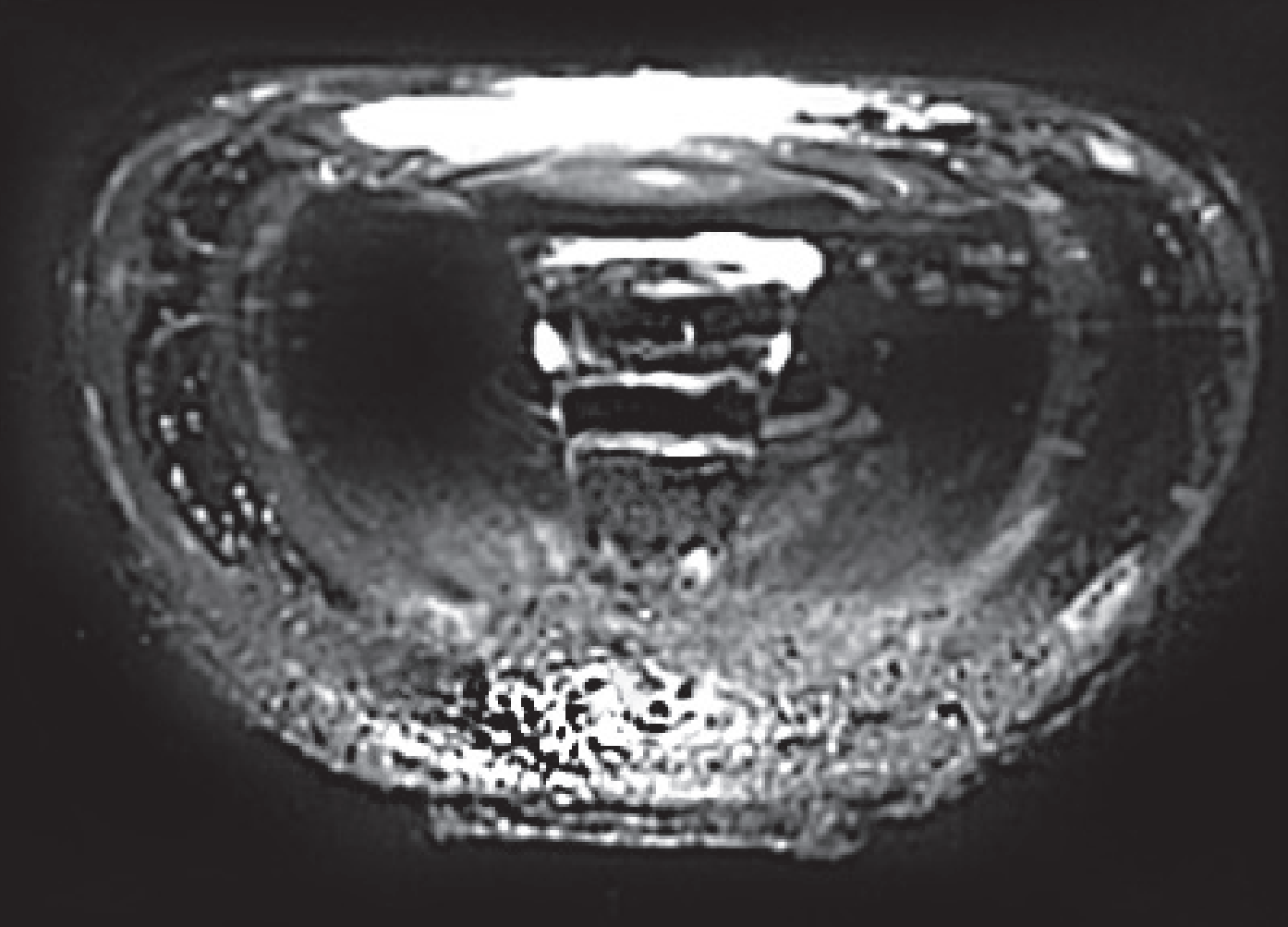

Well before reaching its minimum radius,

2 the collapsing bubble is pierced by the free surface-induced jet. The jet impact causes the first shock wave to be emitted. $\operatorname{Re} \sim 10^{5}$, We $\sim 10^{4}$

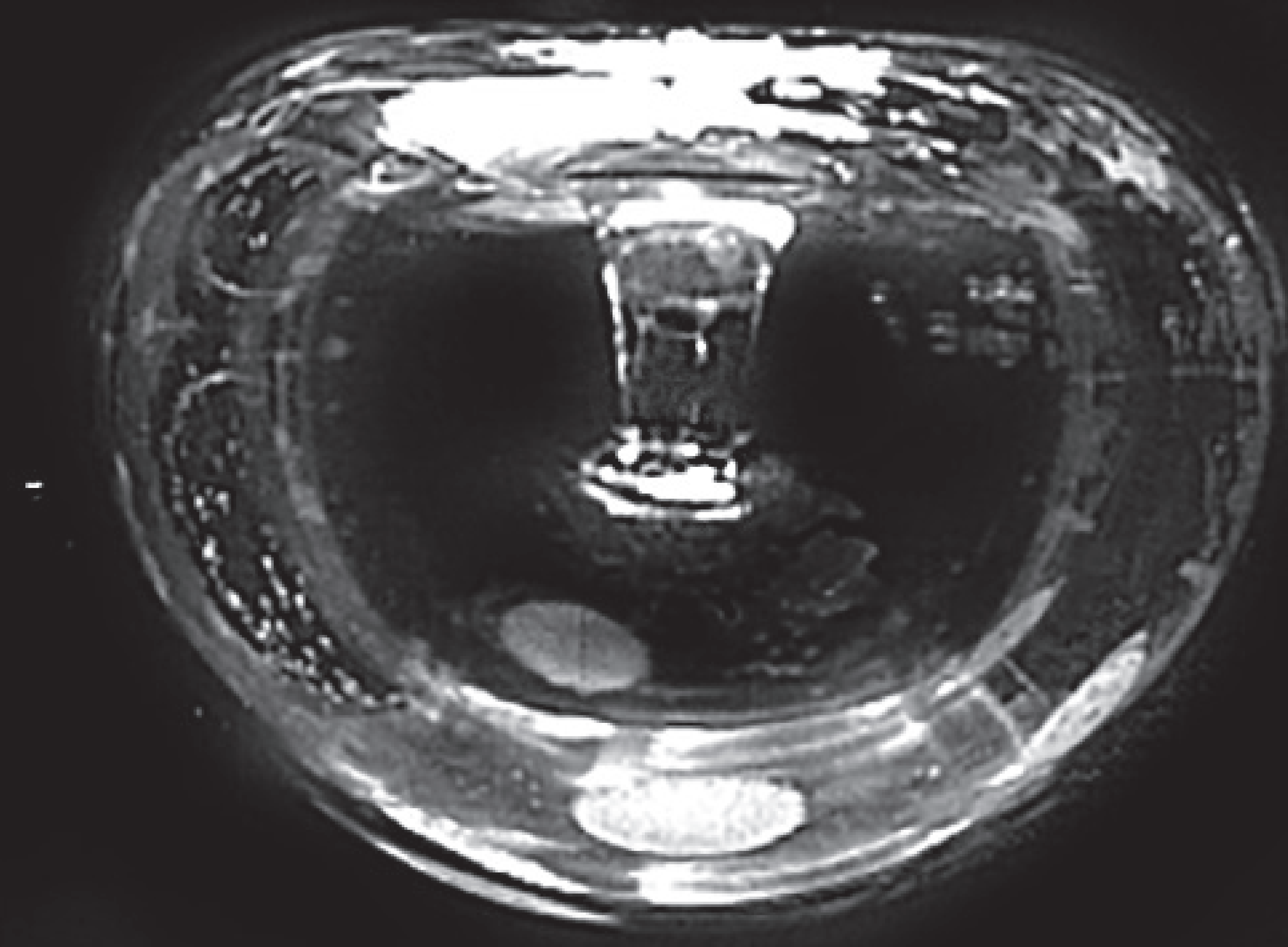

A centimetric bubble is created by means of a focused laser pulse in water nearby a free surface.

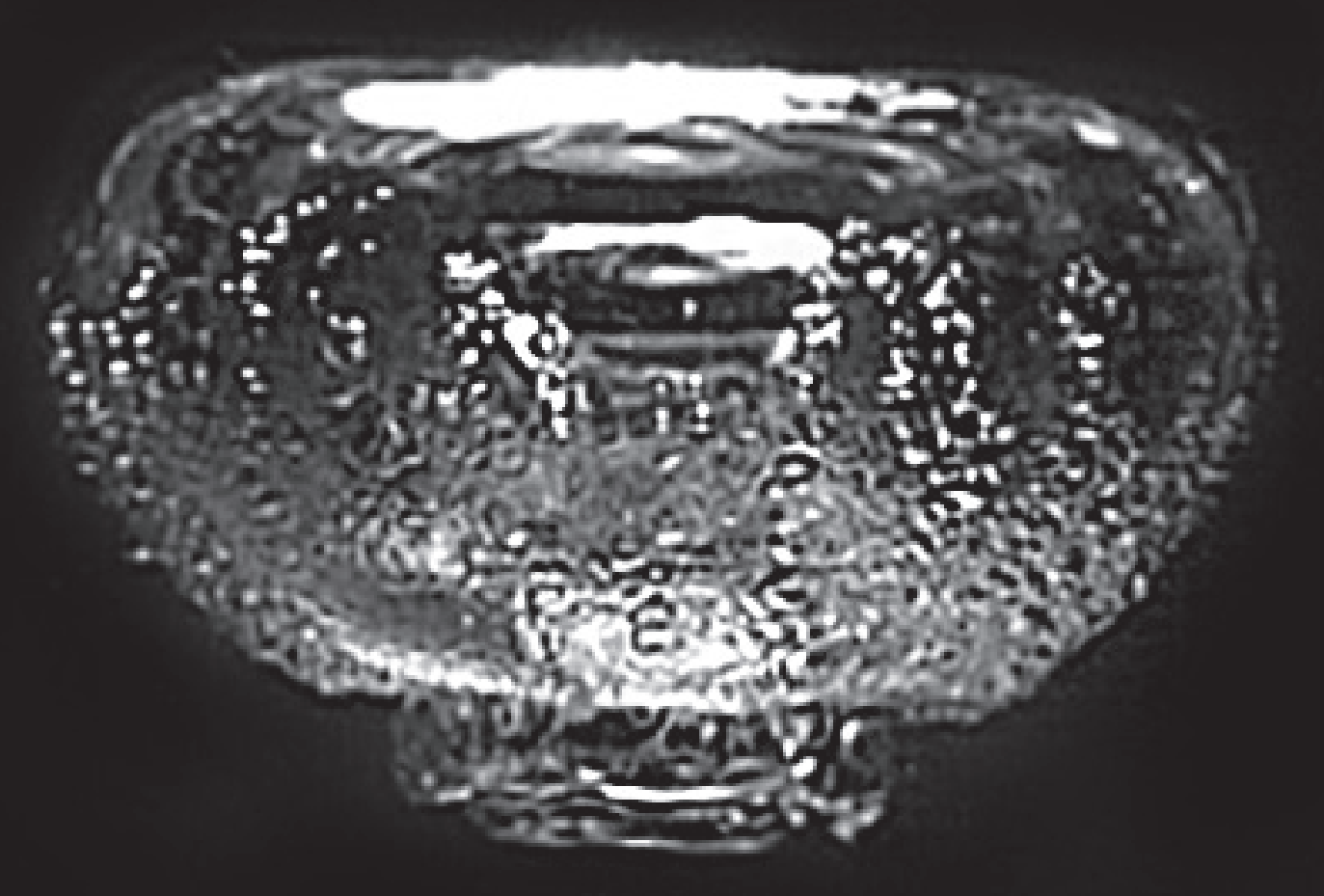

Following the impact, part of the liquid jet propagates along the bubble interface and the rest continues to pierce the wall while entraining a pocket of vapor.

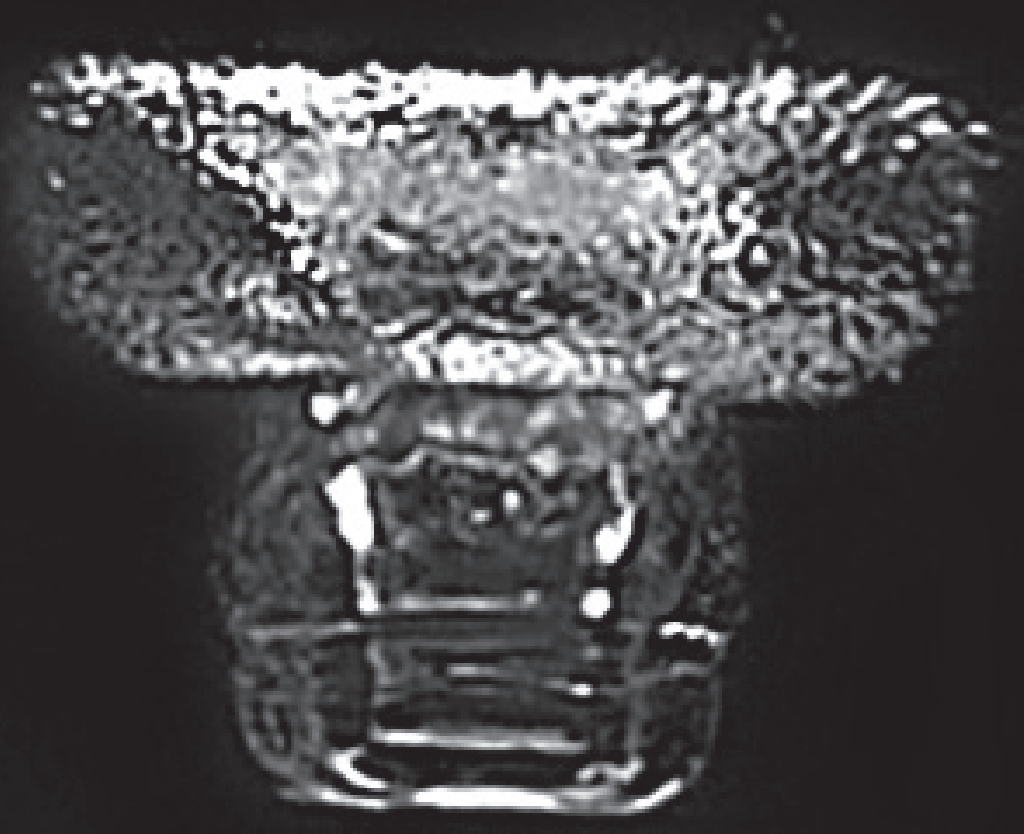

(5) The liquid propagates and ricirculates along the toroidal bubble walls. The bubble collapse is accelerated due to the vapor entrainment.

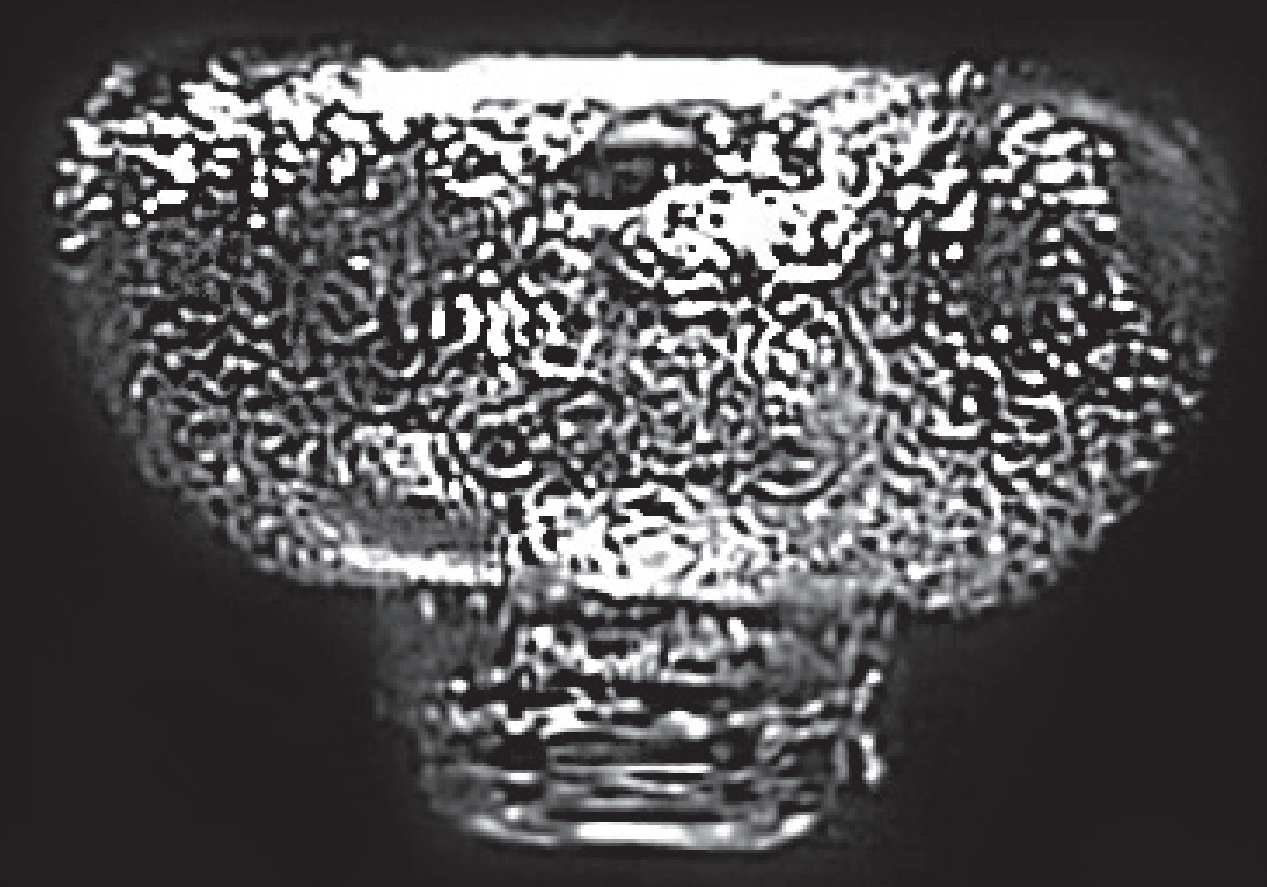

(4) An ejecta-sheet resulting from the jet impact shoots microdroplets onto the bubble wall, resulting in capillary wave formation on the interface.

\section{FNSNF}

